\title{
Revista Colombiana de

\section{Embolización de colaterales en niños con cardiopatías congénitas. Experiencia en un centro cardiovascular}

\author{
Luis F. Carvajal ${ }^{\mathrm{a}, \mathrm{b}, *}$, César O. Bretón ${ }^{\mathrm{a}, \mathrm{b}}$, Margarita M. Zapata ${ }^{\mathrm{b}}$, Mónica I. Guzmán ${ }^{\mathrm{b}}$, \\ Miguel A. Ruz ${ }^{\mathrm{b}}$, Juan R. Donado ${ }^{\mathrm{b}}$, Luis H. Díaz ${ }^{\mathrm{b}}$, Rafael Lince ${ }^{\mathrm{b}}$ y Gloria Franco ${ }^{\mathrm{c}}$
}

a Escuela de Ciencias de la Salud, Universidad Pontificia Bolivariana, Medellín, Colombia
b Servicio de Cardiología Pediátrica, Clínica Cardiovascular Congregación Mariana, Medellín, Colombia
c Sección de Epidemiología, Clínica Cardiovascular Congregación Mariana, Medellín, Colombia

Recibido el 24 de octubre de 2012; aceptado el 2 de mayo de 2013

Disponible en Internet el 22 de agosto de 2014

\author{
PALABRAS CLAVE \\ Embolización; \\ Malformaciones \\ vasculares; \\ Cardiopatía \\ congénita; \\ Cateterismo cardíaco
}

\begin{abstract}
Resumen
Objetivo: Proceder a un análisis descriptivo de los pacientes a quienes se les realizaron embolizaciones de colaterales por vía percutánea.

Materiales y métodos: Se revisaron las historias clínicas de 27 pacientes entre febrero de 2004 y marzo de 2012. Se hace una descripción de las variables analizadas.

Resultados: En el período descrito se efectuó la embolización percutánea de 33 colaterales en 27 pacientes. La edad promedio al momento del procedimiento fue de 53 meses (1-143 meses). Al $68 \%$ se les había realizado previamente cirugía de Glenn o de Fontan, al $8 \%$ fístula BlalockTaussig y al $24 \%$ otros procedimientos quirúrgicos. En el $76 \%$ de los casos el vaso embolizado fue una colateral venovenosa, y en el $24 \%$ restante, una colateral aortopulmonar. Los dispositivos mecánicos fueron los más utilizados (coils y plug vascular). El Amplatzer Vascular Plug se usó para embolizar colaterales de mayor tamaño. Se obtuvo la oclusión inmediata en el $92 \%$ de los casos. No hubo mortalidad asociada con los procedimientos.

Discusión y conclusiones: La embolización de colaterales por vía percutánea es un procedimiento seguro y con alta tasa de eficacia. Los dispositivos utilizados mostraron ser igualmente efectivos.

(c) 2012 Sociedad Colombiana de Cardiología y Cirugía Cardiovascular. Publicado por Elsevier España, S.L.U. Todos los derechos reservados.
\end{abstract}

\footnotetext{
* Autor para correspondencia.

Correo electrónico: Ifkalil@gmail.com (L.F. Carvajal).
} 


\section{KEYWORDS}

Embolization;

Vascular

malformations;

Congenital heart

disease;

Cardiac

catheterization

\section{Embolization of collateral vessels in children with congenital heart disease. Experience in a cardiovascular center}

\begin{abstract}
Objective: To perform a descriptive analysis of patients who underwent percutaneous embolization of collateral vessels.

Materials and methods: We reviewed the medical records of 27 patients between February 2004 and March 2012, and made a description of the variables analyzed.

Results: In the period described, percutaneous embolization of 33 collateral vessels was performed in 27 patients. The mean age at the time of the procedure was 53 months (1-143 months). $68 \%$ had previously undergone Glenn or Fontan surgery, $8 \%$ Blalock-Taussig shunt and $24 \%$ other surgical procedures. In $76 \%$ of cases the vessel embolized was a venovenous collateral and in the remaining $24 \%$ an aortopulmonary collateral. Mechanical devices were most commonly used (coils and vascular plug). The Amplatzer Vascular Plug was used to embolize larger collateral vessels. Immediate occlusion was obtained in $92 \%$ of cases. There was no mortality associated with the procedures.

Discussion and conclusions: Percutaneous embolization is a safe procedure with high success rate. The devices used were shown to be equally effective.

(c) 2012 Sociedad Colombiana de Cardiología y Cirugía Cardiovascular. Published by Elsevier España, S.L.U. All rights reserved.
\end{abstract}

\section{Introducción}

El desarrollo de vasos colaterales es una condición que con frecuencia se observa en pacientes con cardiopatías congénitas; en ocasiones lleva a compromiso hemodinámico y hace necesario su cierre.

En las dos últimas décadas se ha ganado experiencia en el campo de la cardiología pediátrica intervencionista ${ }^{1}$, por lo que los vasos colaterales son susceptibles de cierre por vía percutánea con disponibilidad de varios dispositivos que permiten su oclusión ${ }^{2,3}$.

El objetivo de la investigación fue efectuar un análisis descriptivo de los pacientes a quienes se les realizaron embolizaciones de colaterales por vía percutánea, así como mostrar los resultados inmediatos, las complicaciones y los diferentes dispositivos utilizados.

\section{Materiales y métodos}

Se procedió a un estudio retrospectivo-descriptivo, tipo serie de casos, de una cohorte de pacientes pediátricos con cardiopatías congénitas de base, entre febrero de 2004 y marzo de 2012, a quienes se les realizó embolización de colaterales por vía percutánea en la Clínica CardiovascularCongregación Mariana (Medellín, Colombia).

Se incluyeron todos los pacientes menores de 18 años a quienes se les realizó el procedimiento en mención. Se excluyeron aquellos en quienes no fuera posible obtener la información requerida de las variables de interés: edad, cardiopatía de base, tipo y tamaño de la colateral embolizada, tipo y tamaño del dispositivo utilizado, relación entre el tamaño de la colateral y el dispositivo usado, y resultado obtenido.
Los datos se extrajeron de manera retrospectiva de la base de datos del servicio de hemodinámica pediátrica y de la historia clínica de cada paciente, de donde se recolectó la información de las variables de interés.

Los dispositivos utilizados en esta serie fueron:

- Coils de liberación controlada (Flipper $\left.{ }^{\circledR}\right)$.

- Coils de liberación no controlada $\left(\right.$ Gianturco $\left.{ }^{\circledR}\right)$ : espirales metálicas con filamentos adheridos para aumentar su capacidad oclusora.

- Amplatzer ${ }^{\circledR}$ vascular plug I (St. Jude Medical) (AVP): cilindros autoexpandibles construidos con malla de nitinol.

- Amplatzer VSD membranous ocluder (St. Jude Medical): malla de nitinol rellena en tela de poliéster.

Puesto que no se contaba con un protocolo que determinara el tipo de dispositivo a utilizar, la decisión se tomó con base en las características del vaso a embolizar.

Se consideró oclusión total cuando al realizar la angiografía a los 10 minutos de la liberación del dispositivo el paso del medio de contraste a través de la malla del AVP era mínimo o no se observaba.

Para la revisión de las historias se tuvo en cuenta el respeto por la confidencialidad de los documentos y el cumplimiento previo de las normas del Comité de Ética de la Institución y de la Universidad.

Los resultados de las variables discretas se informaron con frecuencias y porcentajes, y los numéricos, con promedios y desviaciones estándar. Las pruebas chi cuadrado y T de Student se utilizaron para la distribución de probabilidades. Todos los datos fueron analizados con SPSS versión 19 (SPSS Inc.; Chicago, Illinois, EE. UU.). Valores de p iguales o menores de 0,05 fueron considerados estadísticamente significativos. 
Tabla 1 Características de los pacientes y dispositivos utilizados

\begin{tabular}{|c|c|c|c|c|c|c|}
\hline Caso & Edad (meses) & Diagnóstico & $\begin{array}{l}\text { Tipo y tamaño de } \\
\text { la colateral }(\mathrm{mm})\end{array}$ & $\begin{array}{l}\text { Tipo y tamaño del } \\
\text { dispositivo }(\mathrm{mm})\end{array}$ & $\begin{array}{l}\text { Número de } \\
\text { dispositivos }\end{array}$ & Resultado \\
\hline 1 & 36 & $\begin{array}{l}\text { Doble entrada a } \\
\text { ventrículo único, Glenn }\end{array}$ & Colateral vv/4 & AVP/8-10 & 2 & Oclusión total \\
\hline 2 & 72 & Tetralogía de Fallot & Colateral AoP/2,9 & Coils/5 & 2 & $\begin{array}{l}\text { Oclusión } \\
\text { parcial }\end{array}$ \\
\hline 3 & 25 & $\begin{array}{l}\text { Dextrocardia, DCSVD, } \\
\text { canal AV disbalanceado, } \\
\text { CATVP supracardíaca, } \\
\text { Glenn }\end{array}$ & Colateral vv/7 & $\mathrm{AVP} / 14$ & 1 & Oclusión total \\
\hline 4 & 61 & $\begin{array}{l}\text { Atresia pulmonar septum } \\
\text { íntegro, Glenn }\end{array}$ & Colateral vv/4 & $\mathrm{AVP} / 8$ & 1 & Oclusión total \\
\hline 5 & 60 & SVIH, Fontan fenestrado & Colateral vv/2,5 & $\mathrm{AVP} / 4$ & 1 & Oclusión total \\
\hline 6 & 46 & SVIH Norwood Glenn & Colateral vv/4 & AVP/8 & 1 & Oclusión total \\
\hline 7 & 26 & PO CATVP & $\begin{array}{l}\text { Vena vertical } \\
\text { permeable } / 7,7\end{array}$ & $\mathrm{AVP} / 16$ & 1 & Oclusión total \\
\hline 8 & 64 & SVIH Norwood Glenn & Colateral vv/5 & AVP/10 & 1 & Oclusión total \\
\hline 9 & 57 & Atresia tricúspide-Glenn & Colateral vv/5 & AVP/12 & 1 & Oclusión total \\
\hline 10 & 47 & SVIH Norwood Glenn & Colateral vv/5 & $\mathrm{AVP} / 12$ & 1 & Oclusión total \\
\hline 11 & 99 & PO Fontan fenestrado & $\begin{array}{l}\text { Colateral } \\
\text { vv/4,8-6,5 }\end{array}$ & AVP/10-12 & 2 & Oclusión total \\
\hline 12 & 8 & PO CATVP & $\begin{array}{l}\text { Vena } \\
\text { colectora/7,5 }\end{array}$ & $\mathrm{AVP} / 16$ & 1 & Oclusión total \\
\hline 13 & 60 & PO Fontan fenestrado & $\begin{array}{l}\text { Colateral } \\
\mathrm{vv} / 5,9-4,4\end{array}$ & AVP/10-8 & 2 & Oclusión total \\
\hline \multirow[t]{2}{*}{14} & 44 & TCGA Fontan fenestrado & Colateral vv/3,2 & Coils/5-3 & 2 & Oclusión total \\
\hline & & Hipoplasia VD & 4,8 & AVP $/ 8$ & 1 & \\
\hline 15 & 12 & $\begin{array}{l}\text { Dextrocardia ventrículo } \\
\text { único EP mixta severa, } \\
\text { PO Glenn }\end{array}$ & Colateral vv/10,9 & AVP/14 & 1 & Oclusión total \\
\hline 16 & 1 & Secuestro pulmonar, PCA & Colateral AoP/4 & $\mathrm{AVP} / 7$ & 1 & $\begin{array}{l}\text { Oclusión } \\
\text { parcial }\end{array}$ \\
\hline 17 & 5 & $\begin{array}{l}\text { POP Glenn bidireccional, } \\
\text { atresia tricúspide } \\
\text { clásica, ventana } \\
\text { aortopulmonar }\end{array}$ & Colateral vv/1,4 & Coils/5 & 1 & Oclusión total \\
\hline 18 & 22 & $\begin{array}{l}\text { Atresia tricúspide } \\
\text { Tronco común Glenn }\end{array}$ & $\begin{array}{l}\text { Colateral vv/11,8 } \\
11,4\end{array}$ & AVP/16-14 & 2 & $\begin{array}{l}\text { Oclusión } \\
\text { parcial }\end{array}$ \\
\hline 19 & 143 & Criss cross, POP Glenn & $\begin{array}{l}\text { Colateral } \\
v v / 10,1-9,6\end{array}$ & AVP/16-14 & 2 & Oclusión total \\
\hline 20 & 110 & $\begin{array}{l}\text { PO AP con CIV, estenosis } \\
\text { subpulmonar }\end{array}$ & Colateral AoP/3,4 & Coils/8-5 & 2 & Oclusión total \\
\hline 21 & 37 & POP Fontan fenestrado & $\begin{array}{l}\text { Colateral vv y } \\
\text { AoP } / 1,5-3,5\end{array}$ & Coils/3 & 3 & Oclusión total \\
\hline 22 & 40 & POP Fontan fenestrado & Colateral AoP/3 & Coils/3 & 2 & Oclusión total \\
\hline 23 & 80 & $\begin{array}{l}\text { Atresia pulmonar sin CIV, } \\
\text { PO fístula BT }\end{array}$ & Colateral AoP/3,5 & Coils/5 & 2 & Oclusión total \\
\hline 24 & 84 & Fístula AV pulmonar & $\begin{array}{l}\text { Embolización de } \\
\text { fístula AV/8 }\end{array}$ & $\begin{array}{l}\text { Amplatzer VSD } \\
\text { ocluder/14 }\end{array}$ & 1 & Oclusión total \\
\hline 25 & 36 & $\begin{array}{l}\text { Ventrículo único POP } \\
\text { Glenn }\end{array}$ & $\begin{array}{l}\text { Colateral } \\
\text { venovenosa } / 7\end{array}$ & $\mathrm{AVP} / 12$ & 1 & Oclusión total \\
\hline 26 & 36 & $\begin{array}{l}\text { Síndrome de ventrículo } \\
\text { izquierdo hipoplásico, } \\
\text { POP Glenn }\end{array}$ & $\begin{array}{l}\text { Colateral } \\
\text { venovenosa/4 }\end{array}$ & $\mathrm{AVP} / 6$ & 1 & Oclusión total \\
\hline 27 & 156 & $\begin{array}{l}\text { Atresia pulmonar sin CIV, } \\
\text { POP Fontan }\end{array}$ & Colateral AoP/4,2 & Coils/5 & 2 & Oclusión total \\
\hline
\end{tabular}


Tabla 2 Relación entre los dispositivos utilizados y las colaterales

\begin{tabular}{|c|c|c|c|c|c|c|}
\hline \multirow[t]{2}{*}{ Valores registrados } & \multicolumn{2}{|c|}{ Tipos de dispositivos } & \multirow[t]{2}{*}{ T de Student } & \multirow{2}{*}{$\begin{array}{l}\text { Diferencia } \\
\text { de medias }\end{array}$} & \multirow[t]{2}{*}{ IC 95\% } & \multirow[t]{2}{*}{$\mathrm{p}$} \\
\hline & AVP & Coils & & & & \\
\hline $\begin{array}{l}\text { Diámetro de la colateral } \\
(\mathrm{mm}) \text {, promedio }(\mathrm{DE})\end{array}$ & $6,4( \pm 6,3)$ & $3( \pm 0,9)$ & 5,32 & 3,43 & $2,1-4,7$ & 0,000 \\
\hline $\begin{array}{l}\text { Diámetro del dispositivo } \\
\text { en }(\mathrm{mm}) \text {, promedio } \\
\text { (DE) }\end{array}$ & $11( \pm 3,5)$ & $4,4( \pm 1,4)$ & 8,37 & 6,6 & $5,99-8,2$ & 0,000 \\
\hline $\begin{array}{l}\text { Relación entre el tamaño } \\
\text { del dispositivo/colateral } \\
\text { (promedio) }\end{array}$ & 1,81 & 1,84 & $-0,1$ & 0,03 & $-0,76(-0,7)$ & 0,9 \\
\hline
\end{tabular}

\section{Resultados}

En el período descrito se realizó la embolización percutánea de 33 colaterales en 27 pacientes. Se obtuvo información de las variables de interés del total de pacientes incluidos en el análisis.

Las indicaciones para realizar el procedimiento fueron:

- Desaturación: 19 pacientes $(70,3 \%)$.

- Sobrecarga de volumen: 7 pacientes $(26 \%)$.

- Riesgo de sangrado: un paciente $(3,7 \%)$.

La edad promedio al momento del procedimiento fue de 53 meses (1-156 meses); el $59 \%$ fueron hombres y el peso promedio fue de $15,4(2,9-35) \mathrm{kg}$.

Al $40 \%$ se les realizó previamente cirugía de Glenn, al $28 \%$ cirugía de Fontan, al $8 \%$ fístula de Blalock-Taussig y al $24 \%$ otros procedimientos quirúrgicos (tabla 1).

Se embolizó la colateral venovenosa en el $74 \%$ de los casos y la colateral aortopulmonar en el $26 \%$ restante (tabla 1$)$.

Se utilizó AVP en 19 pacientes, coils (Gianturco ${ }^{\circledR}$ ) (Cook Medical) en 7, detachable embolization coils (Flipper ${ }^{\circledR}$ ) (Cook Medical) en uno, y Amplatzer ${ }^{\circledR}$ VSD ocluder (St. Jude Medical) en uno (tabla 1).

El acceso vascular se realizó por vía femoral en 11 pacientes $(40,7 \%)$, por vía yugular en $2(7,4 \%)$ y por ambas vías en $14(51,9 \%)$.

La elección del acceso vascular dependía de la anatomía y de la localización del vaso a embolizar y de la preferencia del grupo de hemodinámica.

Se utilizó el AVP para embolizar las colaterales de mayor tamaño. No hubo diferencia estadísticamente significativa entre el diámetro del dispositivo y de la colateral embolizada al comparar AVP y coils (tabla 2).
Al confrontar el número de dispositivos utilizados para el cierre de colaterales, se encontró que con el empleo de coils se requieren más dispositivos que cuando se utilizan AVP, siendo esta diferencia significativa (tabla 3).

Se obtuvo oclusión total en el $92 \%$ de los casos. En quienes se indicó embolización por desaturación se evidenció mejoría inmediata en la pulso-oximetría luego de realizar la oclusión de la colateral.

No hubo mortalidad asociada a los procedimientos y solo se registró una complicación (disección de la arteria femoral).

\section{Discusión}

El tratamiento de pacientes con cardiopatías que requieren corrección univentricular o aquellos sometidos a períodos largos de hipoxemia ha hecho que los cardiólogos pediatras se enfrenten con más frecuencia a pacientes que desarrollan vasos colaterales. Es así como los pacientes con cirugía de Glenn o Fontan, debido a sus patrones de circulación y a los cambios de presión en el sistema circulatorio, están predispuestos a desarrollar vasos colaterales, los cuales pueden llevar a desaturación. Igualmente, en pacientes con otras cardiopatías congénitas las colaterales pueden generar cortocircuitos con sobrecarga de volumen e importante repercusión hemodinámica, que requerirá algún tipo de intervención ${ }^{4,5}$.

En este panorama, la embolización percutánea ha demostrado ser un método seguro y efectivo para lograr oclusiones vasculares $^{6,7}$. En el campo de la cardiología pediátrica intervencionista se ha logrado adquirir mayor experiencia en los últimos años y, junto con el advenimiento de nuevos dispositivos, se ha favorecido este crecimiento $^{8-13}$.

Tabla 3 Relación entre el tipo y el número de dispositivos utilizados

\begin{tabular}{|c|c|c|c|c|}
\hline \multirow[t]{2}{*}{ Dispositivo utilizado } & \multicolumn{2}{|c|}{$\begin{array}{l}\text { Número de dispositivos utilizados por } \\
\text { colateral embolizada }\end{array}$} & \multirow[t]{2}{*}{ Chi cuadrado } & \multirow[t]{2}{*}{$\mathrm{p}$} \\
\hline & Dos o más & Uno & & \\
\hline Uso de coil & 7 & 2 & 18,6 & 0,0001 \\
\hline Uso de AVP & 1 & 22 & & \\
\hline
\end{tabular}


En la actualidad se cuenta con un sinnúmero de dispositivos y materiales para la oclusión de vasos sanguíneos según el escenario clínico que se presente.

Ahora se conocen diferentes grupos de dispositivos, tales como:

- Agentes líquidos: n-butil-cianoacrilato, Onyx.

- Partículas: gelfoam, colágeno microfibrilar, alcohol polivinilo, microesferas.

- Coils.

- Plugs.

- Balones ${ }^{2,6}$.

En general, los coils, plugs y balones se utilizan para ocluir grandes vasos, mientras que los líquidos y partículas se emplean para ocluir pequeñas arterias, arteriolas y redes capilares $^{2,6}$.

En la serie del estudio, los dispositivos más utilizados fueron mecánicos (coils y plug vasculares); los coils se utilizaron en los vasos de menor calibre y los AVP en los de mayor calibre, tal como lo reportado por Girona et al. ${ }^{5}$. Estos dispositivos son más económicos y fáciles de usar; sin embargo, en vasos de calibre superior se requiere mayor número de dispositivos, hecho que incrementa los costos; así mismo, el procedimiento toma más tiempo y existe mayor riesgo de desplazamiento del dispositivo ${ }^{5,14,15}$. Por su parte, los AVP están disponibles para uso a partir de 2004, y desde entonces se han desarrollado varios tipos, entre los que ahora se cuentan del tipo I al IV. El AVP utilizado en esta serie fue el I. Estos dispositivos son seguros, se cuenta con variedad de diámetros que permiten su uso en vasos de diverso calibre y se pueden remover con relativa facilidad cuando el tamaño o la posición no sean adecuados. No obstante, su costo es mayor cuando se compara con los coils para vasos pequeños, si bien dicha diferencia deja de ser significativa cuando se requieren múltiples coils ${ }^{14,16}$.

En la serie expuesta, en la mayoría de casos en donde se utilizaron coils fue necesario emplear más de un dispositivo, a diferencia de los casos en los que se utilizó el AVP, en quienes en el $95 \%$ de los casos se requirió solo un dispositivo para ocluir la colateral. Ello cobra relevancia a la hora de decidir qué dispositivo se debe emplear. Las casas fabricantes de ambos dispositivos recomiendan utilizar referencias con diámetros $30-50 \%$ mayores que el del vaso a embolizar.

De otra parte, en términos generales, las complicaciones asociadas con los procedimientos son escasas. La más descrita con el uso de coils es la embolización del dispositivo, con incidencias reportadas de hasta el $4 \%^{7}$; sin embargo, con el advenimiento de los sistemas de liberación controlada estas cifras se han reducido $0^{7,15,17,18}$. En esta serie, la única complicación descrita fue la disección de la arteria femoral en un paciente de un mes de edad, con un peso de $2,9 \mathrm{~kg}$, a quien se le embolizó una colateral aortopulmonar de $4 \mathrm{~mm}$ con un AVP de $7 \mathrm{~mm}$. Una de las desventajas que tiene el AVP tipo I es la necesidad de requerir catéteres de $5 \mathrm{Fr}$ como mínimo, lo cual limita su uso en niños más pequeños. No obstante, con los nuevos AVP se supera este inconveniente, ya que se pueden utilizar catéteres $4 \mathrm{Fr}$.

En cuanto a la capacidad oclusora de los dispositivos utilizados, evaluada por angiografía, se presentó oclusión parcial en el $9 \%(n=3)$ de los casos, sin encontrar diferencias estadísticamente significativas entre los dispositivos utilizados, similar a lo descrito en otras series ${ }^{5,14}$. Solo en uno de esos casos la relación entre el tamaño del dispositivo y el de la colateral fue menor de $1,3 \mathrm{~mm}$.

\section{Limitaciones del estudio}

Pese a que se trata de un estudio de tipo retrospectivo con las limitaciones que esto implica, principalmente en cuanto a recolección de la información y calidad de la misma, se pudieron obtener todos los datos de interés requeridos con base en el objetivo propuesto al inicio de la investigación, lo cual minimizó el sesgo de información.

\section{Conclusiones}

La embolización de colaterales por vía percutánea en niños con cardiopatías congénitas es un procedimiento seguro y con alta tasa de eficacia. Los dispositivos utilizados mostraron ser igualmente efectivos; no obstante, la elección de uno u otro dependerá del tamaño del vaso a embolizar.

\section{Financiación}

Los autores manifiestan no haber recibido apoyo financiero para la investigación y/o la autoría de este artículo.

\section{Conflicto de intereses}

Los autores declaran no tener conflicto de intereses.

\section{Bibliografía}

1. Beekman R, Shim D, Lloyd T. Embolization therapy in pediatric cardiology. J Interv Cardiol. 1995;8:543-56.

2. Walsh K. Advanced embolization techniques. Pediatr Cardiol. 2005;26:275-88.

3. Xie $\mathrm{CH}$, Xia CH, Gong F, Zhou Y, Zhu W. Interventional occlusion of congenital vascular malformations. World J Pediatr. 2009;5:296-9.

4. Andrews R, Tulloh R, Anderson D. Coil occlusion of systemic venous collaterals in hypoplastic left heart syndrome. Heart. 2002;88:167-9.

5. Girona J, Martí G, Betrían P, Ferrán G, Jaume C. Embolización percutánea de fístulas vasculares con el tapón vascular de Amplatzer o coils. Rev Esp Cardiol. 2009;62:765-73.

6. Sim J, Alejos A, Moore J. Techniques and applications of transcatheter embolization procedures in pediatric cardiology. Interven Cardiol. 2003;16:425-48.

7. Tan C, Levi D, Moore J. Embolization and transcatheter retrieval of coils and devices. Pediatr Cardiol. 2005;26:267-74.

8. Schwartz M, Glatz A, Rome J, Gillespie M. The Amplatzer vascular plug and Amplatzer vascular plug II for vascular occlusion procedures in 50 patients with congenital cardiovascular disease. Catheter Cardiovasc Interv. 2010;76:411-7.

9. Fuhrman B, Bass J, Castaneda-Zuniga W, Amplatz K, Lock J. Coil embolization of congenital thoracic vascular anomalies in infants and children. Circulation. 1984;70:285-9.

10. Perry S, Radtke W, Fellows K, Keane J, Lock J. Coil embolization to occlude aorto pulmonary collateral vessels and shunts in patients with congenital heart disease. J Am Coll Cardiol. 1989;13:100-8. 
11. Orsmond G. Coil embolization in congenital heart disease. Prog Pediatr Cardiol. 1992;1:44-54.

12. Rothman A, Tong A. Percutaneous coil embolization of superfluous vascular connections in patients with congenital heart disease. Am Heart J. 1993;126:206-13.

13. Moore J, Berdjis F. Coil occlusion of congenital vascular malformation and surgical shunts. Prog Pediatr Cardiol. 1996;6:149-59.

14. Barbaros C,, Bora P, Murat C, Erhan A, Serdar G, Türkmen Ç. Peripheral vascular applications of the Amplatzer $^{\circledR}$ vascular plug. Diagn Interv Radiol. 2008;14:35-9.

15. Sato $Y$, Ogino $H$, Hara $M$, Satake $M$, Oshima $H$, Banno $T$, et al. Embolization of collateral vessels using mechanically detachable coils in young children with congenital heart disease. Cardiovasc Intervent Radiol. 2003;26:528-33.

16. Farra H, Balzer DT. Transcatheter occlusion of a large pulmonary arteriovenous malformation using the Amplatzer vascular plug. Pediatr Cardiol. 2005;26:683-5.

17. Huggon C, Qureshi S, Reidy J, Dos Anjos R, Baker E, Tynan M. Percutaneous transcatheter retrieval of misplaced therapeutic embolisation devices. Br Heart J. 1994;72: 470-5.

18. Prieto L, Latson L, Dalvi B, Arbetman M, Ebeid M, Lamorgese T. Transcatheter coil embolization of abnormal vascular connections using a new type of delivery catheter for enhanced control. Am J Cardiol. 1999;83:981-3. 